\title{
NUEVAS PERSPECTIVAS EN EL TRATAMIENTO JURISPRUDENCIAL DE LA CLEPTOMANÍA ${ }^{1}$
}

\author{
María Sánchez Vilanova \\ Departamento Derecho Penal / Instituto Universitario de Investigación en \\ Criminología y Ciencias Penales (ICCP) \\ Universidad de Valencia
}

Resumen: El presente trabajo pretende acercarse a la realidad forense de la cleptomanía desde una óptica multidisciplinar. Por ello, nos aproximaremos a este trastorno del control de los impulsos, con clara incidencia en la imputabilidad, tanto desde una perspectiva clínica, centrada en los últimos avances neurobiológicos al respecto, como legal, presentando un estudio jurisprudencial y ahondando en sus características definitorias para facilitar un mejor tratamiento jurídico.

Palabras Clave: cleptomanía - imputabilidad - eximente - control impulsividad

Abstract: This paper aims to approach to forensic reality about kleptomania from a multidisciplinary perspective. Thus, it approaches the disorder of impulse control, with clear impact on imputability, both from a clinical perspective, focusing on its latest neurobiological developments, and legal perspective, introducing a jurisprudential study and deepening into its defining features to facilitate a better legal treatment.

Keywords: kleptomania - imputability - eximente - control - impulsividad

1 Este trabajo ha sido elaborado en el marco de los Proyectos de investigación "Derecho penal de la peligrosidad: tutela y garantía de los derechos fundamentales" (DER2017-86336-R), financiado por la Agencia Estatal de Investigación y el Fondo Europeo de Desarrollo Regional (FEDER) (IP: Lucía Martínez Garay) y "Derecho penal y comportamiento humano» (MICINN-RTI2018-097838-B-100), concedido por el Ministerio de Ciencia, Innovación y Universidades de España (IP: Eduardo Demetrio Crespo). 


\section{CUESTIONES PREVIAS}

La cleptomanía es una entidad psicopatológica que se ha englobado tradicionalmente dentro del espectro de los trastornos del control de los impulsos (en adelante, TCI). No obstante, la misma no es ejemplificativa de la escasa trascendencia legal que este grupo de perturbaciones ostenta. Si bien los TCI son diagnosticados y sufridos por un importante número de personas, los mismos, en general, no comportan la comisión de comportamientos delictivos ${ }^{2}$. Ahora bien, aunque la prevalencia de la cleptomanía es poco conocida, situándose en torno a 1 de cada 1.000 personas, en el ámbito penal es un trastorno que, como seguidamente se abordará, debe ser convenientemente considerado, en atención a su repercusión en la imputabilidad de los sujetos que la padecen, y su consiguiente merma en la responsabilidad.

De entrada, se puede destacar que nos encontramos ante un trastorno en el que los sujetos, repetidamente, son incapaces de resistir el impulso que sienten de robar objetos que no los usan, ni de forma personal, ni los destinan a fines lucrativos. Este es, sin duda, un aspecto de capital importancia en la cleptomanía, puesto que los objetos que estas personas sustraen no son necesarias para ellas, por lo que es frecuente que, poco después de la comisión de los hechos delictivos, se deshagan de los mismos o los regalen ${ }^{3}$, explicándose por tanto su actuación como resultado del denominado «impulso irresistible», un concepto verdaderamente controvertido y cuestionado que es difícilmente comprensible por la jurisprudencia. Ciertamente, aunque es de sobra conocido que los TCI

2 Las clasificaciones internacionales de los trastornos mentales (concretamente, la clasificación internacional de enfermedades mentales, conocida como CIE, y el manual diagnóstico y estadístico de los trastornos mentales, nombrado por sus siglas DSM), se refieren a la cleptomanía como uno de los trastornos más representativos de los TCI, a los que se tiene que añadir la piromanía, la ludopatía, la tricotilomanía, y el trastorno explosivo intermitente. No obstante, mientras que la cleptomanía o la piromanía pueden llevar a las personas que los padecen a la comisión de actos criminales, en la mayoría de ellos esto no es frecuente, pues los casos en los que los ludópatas o aquellos con trastorno explosivo intermitente cometen actos delictivos como consecuencia de su trastorno son excepcionales. No en balde, DELGADO BUENO y RODRÍGUEZ MARTOS DAUER han destacado como la clasificación conjunta de estas tipologías se explica, únicamente, en base a la similitud en sus características definitorias. Conviene apuntar, por último, que pese a ser los subtipos de estos trastornos similares en ambas clasificaciones internacionales, en los últimos años se ha producido una modificación al respecto, pues el DSM, en su quinta edición, la cual ejemplifica aun giro hacia un modelo dimensional, incluye el trastorno antisocial de la personalidad como un subtipo de los TCI.

3 IBÁÑEZ CUADRADO, A., y SAIZ RUIZ, J.: «Trastornos de los hábitos y del control de los impulsos», en Tratado de Psiquiatría, cap. 32, p. 579 - 602. 
conllevan un menoscabo en la capacidad de las personas de control o represión de los impulsos (e inclusive en algunas ocasiones pueden llegar a realizar conductas en contra de su voluntad), la etiología desconocida de estos trastornos impide que los mismos sean tomados en consideración en numerosas ocasiones. Al margen de ello, se constata que esta perturbación muestra sus primeros síntomas a edades tempranas, siendo la misma crónica y, como en el análisis que en el punto tercero se presenta, frecuentemente femenina ${ }^{4}$.

Respecto de sus características clínicas, cabe destacar, previamente, y sin entrar en el estudio en profundidad del trastorno, efectuado en el siguiente epígrafe, que los afectados por el mismo sufren una tensión o activación interior irresistible, como consecuencia de la cual cometen pequeños hurtos, a causa de una importante merma en su capacidad de control, si bien conservan el conocimiento respecto de su actuación ${ }^{5}$. Así pues, su incidencia en la imputabilidad es manifiesta, dada la importante limitación en la capacidad inhibitoria de las personas que estos trastornos provocan. Por tanto, podrían llevar aparejados, en función de la intensidad del trastorno en las facultades cognitivas de los sujetos que lo padecen y su relación con la actuación delictiva, la apreciación tanto de la eximente incompleta o la circunstancia atenuante analógica, como también una eximente completa. En cualquier caso, como en el análisis jurisprudencial se tendrá ocasión de comprobar, no existe abundante jurisprudencia sobre estos trastornos, especialmente del Tribunal Supremo (en adelante, TS), puesto que las infracciones por las que, de normal, estos sujetos son detenidos y procesados, difícilmente alcanzan la casación.

Se debe tener en cuenta que los robos perpetrados por los cleptómanos no están justificados ni por la necesidad ni por los deseos de perjudicar a la víctima. De hecho, el robar es un acto solitario y no planeado que les provoca una preocupación importante ante la posibilidad de su detención, lo que lleva a la aparición de signos de ansiedad y depresión, sintiéndose culpables y avergonzados de su conducta. Por tanto, aunque la capacidad cognoscitiva de estos sujetos se mantendría intacta, el control sobre sus impulsos quedaría gravemente mermado. Esto es, cuando un cleptómano realiza una conducta delictiva dominado por un impulso irresistible o irrefrenable de cometer la acción antijurídica para aliviar la tensión insoportable que padece, podría ser de aplicación el artículo (en adelante, art.) 20.1 del Código Penal (en adelante, CP), apreciándose la semieximente del art. 21.1 CP cuando dicha alteración es importante, pero sin llegar a anular la voluntad. Finalmente, cuando el sujeto sólo

4 GISBERT CALABUIG, J.A., SÁNCHEZ BLANQUE, A. y CASTELLANO ARROYO, M.: «Neurosis», en GISBERT CALABUIG, J.A: Medicina Legal y Toxicología, 6 a Edición, Masson, 2004, p. 1179.

5 HOMS SANZ DE LA GARZA, J.: Avances en medicina legal: Ingeniería genética, alteraciones psiquicas y drogas, J.M. Bosch, 1999, p. 53. 
presente una leve disminución en su capacidad para actuar conforme a la comprensión de los hechos realizados, la atenuante analógica del art. 21.6 CP sería de aplicación preferente.

De todos modos, las controversias que la categoría de la imputabilidad presenta dificulta la aplicación de la eximente del artículo $20.1 \mathrm{CP}$ en este trastorno, tal vez debido a la confusión que envuelve a esta misma categoría, la imputabilidad ${ }^{6}$, una de las figuras cuyos contornos es más difícil concretar. Esto es debido, en parte, a la misma complejidad que la categoría general en la que esta se engloba, la culpabilidad, comporta, cuestionándose por la doctrina inclusive su mismo fundamento ${ }^{7}$.

Basta recordar que la exigencia de responsabilidad penal requiere la comprobación ex ante de la culpabilidad del sujeto, definida por COBO DEL ROSAL y VIVES ANTÓN ${ }^{8}$, como el «reproche personal» dirigido al autor por la realización de un hecho típicamente antijurídico. Y, en este juicio, según doctrina general, se ha de acreditar que el sujeto en cuestión presenta una aptitud psíquica y biológica adecuada para conocer la lesividad e ilicitud de su conducta, y dirigir su voluntad conforme a dicha comprensión. Es decir, se ha de constatar su imputabilidad.

Ahora bien, el CP de 1995 no hace mención expresa a la imputabilidad, sino que alude a la falta excepcional de ésta. En realidad, el CP presenta una regulación a sensu contrario de la imputabilidad, refiriéndose en el artículo 20 a los supuestos de ausencia de esta. Concretamente, en su primer apartado declara exento de responsabilidad penal a aquellos que, al que al tiempo de cometer la infracción penal, a causa de cualquier anomalía o alteración psíquica, no puedan comprender la ilicitud del hecho o actuar conforme a esa comprensión ${ }^{9}$. Conforme a esta norma, se extraen los dos requisitos exigibles en esta figura: el requisito cognitivo,

6 Cobo Del ROSAL, M. y VIVES ANTÓN, T.S.: Derecho Penal. Parte general, $5^{\mathrm{a}}$ ed. corregida, aumentada y actualizada, Tirant lo Blanch, Valencia, 1999, p. 576; MORALES PRATS, F. (coordinador) y OTROS: Comentarios al nuevo Código Penal, $4^{\mathrm{a}}$ ed., ThomsonAranzadi, Navarra, 2005, p. 148; GIMBERNAT ORDEIG, E.: ¿Tiene un futuro la Dogmática jurídico-penal?, en Estudios de Derecho Penal, $3^{\mathrm{a}}$ ed., Madrid, Tecnos, 1990, p. 157; MIR PUIG, S.: Derecho Penal. Parte General, $7^{\text {a }}$ ed., $3^{\text {a }}$ reimpr., Reppertor, Barcelona, 2006, p. 558; MUÑOZ CONDE, F. y GARCÍA ARÁN, M.: Derecho Penal. Parte General, $6^{a}$ ed., rev. y puesta al día, Tirant lo Blanch, Valencia, 2004, p. 372, URRUELA MORA, A.: Imputabilidad penal y anomalía o alteración psíquica. La capacidad de culpabilidad penal a la luz de los modernos avances en psiquiatría y genética, Comares, Bilbao-Granada, 2004, p. 156.

7 MARTÍNEZ GARAY, L. La imputabilidad penal. Concepto, fundamento, naturaleza jurídica y elementos, Valencia, Tirant lo Blanch, 2005, p. 15.

8 COBO DEL ROSAL, M. y VIVES ANTÓN, T.S.: p. 535.

9 En el segundo apartado regula el estado de intoxicación plena por el consumo de bebidas alcohólicas, drogas tóxicas, estupefacientes, sustancias psicotrópicas u otras que produzcan efectos análogos, y la alteración en la percepción desde el nacimiento o desde la infancia que produzca una alteración grave de la conciencia de la realidad en el tercero. La minoría de edad, en cambio, la encontramos en el artículo 19 del CP. 
que requiere la capacidad cognitiva del sujeto respecto de la ilicitud de su conducta, y un requisito volitivo o de determinación, que le permita actuar conforme a dicha comprensión, que es precisamente la que se vería comprometida en la cleptomanía.

En base a esta configuración, se observa claramente como la imputabilidad responde a una fórmula mixta; un criterio mixto, refrendado por la jurisprudencia del $\mathrm{TS}^{10}$, en el que no resulta suficiente la existencia de un diagnóstico clínico para apreciar esta figura. El sistema mixto del $\mathrm{CP}$ requiere, además de una causa biopatológica, un efecto psicológico: que esta anomalía anule o afecte gravemente la capacidad de comprender la ilicitud del hecho o de determinar el comportamiento con arreglo a esa comprensión. Por ello, es imprescindible el efecto psicológico en los casos de anomalías o alteraciones psíquicas, pues la enfermedad es condición necesaria pero no suficiente para establecer una relación causal entre la enfermedad mental y el acto delictivo ${ }^{11}$. Como en la STS 175/2008 se declara expresamente, "cuando el autor del delito padezca cualquier anomalía o alteración psíquica, no es tanto su capacidad general de entender y querer, sino su capacidad de comprender la ilicitud del hecho y de actuar conforme a esa comprensión aquello a valorar ${ }^{12}$ ». Y, como destaca MIR PUIG ${ }^{13}$, estos dos requisitos acuñados legalmente, requieren determinados datos biológicos que, en el presente estudio van a ser abordados detenidamente.

En detalle, según la doctrina y la jurisprudencia, la valoración de la imputabilidad debe atender a cuatro criterios: cualitativo (elemento biológico referente a la naturaleza de la perturbación), cuantitativo (elemento psicológico o normativo referente a la intensidad y grado de la perturbación), cronológico (duración del trastorno y permanencia del mismo en el momento de la comisión del hecho delictivo), y la relación de causalidad entre el trastorno y el hecho delictivo ${ }^{14}$, especialmente relevantes en la cleptomanía. Y, sin duda, en relación al cualitativo, se estima que, para no complicar las cosas, todo informe pericial debe tener en sus conclusiones el diagnóstico en base a las clasificaciones internacionales de enfermedades mentales, la CIE o el DSM, no siendo recomendable utilizar terminología que no esté enmarcada en estos códigos ${ }^{15}$. Conviene aclarar que las diferencias entre ambas clasificaciones son irrelevantes; el DSM-V presenta una estructura multiaxial y sólo contempla las enfer-

10 STS 1400/99 de 9 de octubre.

11 STS 314/2005, de 9 de marzo.

12 STS 175/2008, de 14 de mayo.

13 MIR PUIG, S.: p. 556 - 557.

14 ECHEBURÚA ODRIOZOLA, E., AMOR ANDRÉS, P.J. y YUSTE GARCÍA, J.: «Atenuación de la responsabilidad penal en la ludopatía: Bases psicopatológicas», en Psicopatología Clínica Legal y Forense, Vol. 1, $N^{o}$ 0, 2000, p. $59-76$.

15 A efectos informativos, vamos a señalar brevemente las características de estos manuales: 
medades mentales, mientras que la CIE-10 abarca todas las enfermedades y sólo en su capítulo V se valoran las enfermedades mentales.

No obstante, teniendo en cuenta su mayor uso por nuestros tribunales, se abordará la cleptomanía de acuerdo con la quinta versión del $\mathrm{DSM}^{16}$. Más aun si se tiene en cuenta que la nueva versión del DSM representa una tímida apuesta por la regulación dimensional que, como en las conclusiones del presente artículo se tendrá ocasión de comprobar, puede ser más idónea en la regulación de los trastornos mentales, especialmente en el terreno forense, donde más allá de la concreta categoría diagnostica, aquello más importante es la afectación de la capacidad racional del sujeto de la que depende su imputabilidad y, por ende, responsabilidad penal.

\section{ADELANTOS NEUROBIOLÓGICOS EN EL ESTUDIO DE LA CLEPTOMANÍA}

\section{Punto de partida}

A principios del siglo XIX, Esquirol introdujo el término monomanía instintiva para describir un grupo de trastornos del comportamiento que se caracterizaban por la realización de actos impulsivos sin motivo aparente como respuesta a un impulso irresistible ${ }^{17}$. Fue el clásico alienista francés Matías el primero en describir científicamente este trastorno, entendiendo que en esta el control volitivo estaba profundamente comprometido. A pesar de estos precedentes, los TCI no serán reconocidos como enfermedades mentales hasta la década de los ochenta del pasado siglo, cuando la APA las recogerá por primera vez en el DSM-III ${ }^{18}$. Y, en

a) DSM-V. El Manual diagnóstico y estadístico de los trastornos mentales, se trata de un Manual elaborado por el comité de la Asociación de Psiquiatría Americana (APA), en consenso con infinidad de psiquiatras de todo el mundo

b) CIE 10. Clasificación de los Trastornos mentales y del Comportamiento, también surge del consenso entre multitud de médicos, siendo una publicación de la Organización Mundial de la Salud. (OMS).

16 AMERICAN PSYCHIATRIC ASSOCIATION: Diagnostic and Statistical Manual of Mental Disorders (DSM-V), 2014. Podemos observar un interesante análisis de las modificaciones que el borrador del DSM-V, publicado el 19 de febrero de 2010 en la web de la APA, implicaba respecto de la regulación del DSM-IV en los trastornos de la personalidad (muchas de ellas finalmente descartadas en el manual finalmente aprobado) en: ESBEC, E. y ECHEBURRUA, E.: «La reformulación de los trastornos de la personalidad en el DSM-V», en Actas Esp Psiquiatría, 39 (1), 2011, pp. 1-11.

17 McELROY, S.L., KECK, P.E. Jr., POPE, H.G. Jr., HUDSON, J.I., FAEDDA, G.L. y SWANN, A.C.: «Clinical and research implications of the diagnosis of dysphoric or mixed mania or hypomania», en Am J Psychiatry. 1992 Dec; 149(12):1633-44.

18 AMERICAN PSYCHIATRIC ASSOCIATION: Diagnostic and Statistical Manual of Mental Disorders (DSM-III), 1980. 
efecto, su descripción no se aleja mucho de la acientífica descripción del francés.

Los TCI, dentro de los cuales encontramos a la cleptomanía, son un tipo de desórdenes psiquiátricos caracterizados por la impulsividad. Esto es, por su tendencia a la realización de actuaciones en cortocircuito y la ejecución de actos repetidos sin un motivo racional claro que perjudican los intereses del mismo sujeto y de las personas de su entorno ${ }^{19}$.

En la mayor parte de los casos, los sujetos afectados por estos trastornos perciben una sensación de tensión interior justo antes de realizar el acto en cuestión, seguido de una sensación inmediata de placer o gratificación. Tras este, es común el arrepentimiento o el sentimiento de culpa, aunque no siempre se produce. Tal vez ello se deba a que, bajo este concepto integrador, los TCI son un grupo heterogéneo de entidades que conllevan importantes disfunciones personales y sociales, y presentan una gran comorbilidad con otros trastornos psiquiátricos ${ }^{20}$, como seguidamente veremos.

\section{Diagnóstico clínico}

De acuerdo con la nueva regulación del DSM-V, la cleptomanía [312.32 (F63.2 $\left.\left.{ }^{21}\right)\right]$ se diagnostica de acuerdo con los siguientes criterios:

- el fracaso recurrente para resistir el impulso de robar objetos que no son necesarios para uso personal ni por su valor monetario (criterio A)

- el aumento de la sensación de tensión inmediatamente antes de cometer el robo (criterio B)

- placer, gratificación o alivio en el momento de cometerlo (criterio C)

- y que el robo no se cometa para expresar rabia ni venganza, ni en respuesta a un delirio o una alucinación (criterio D), o se explique

19 CABRERA FORNEIRO, J. y FUERTES ROCAÑIN, J.C.: La Salud Mental en los Tribunales. Manual de Psiquiatría Forense y Deontología Profesional, $2^{\mathrm{a}}$ ed. revisada y ampliada, Arán, Madrid, 2007, p. 194.

20 RONCERO, C., RODRÍGUEZ-URRUTIA, A., GRAU-LÓPEZ, L., CASAS, M.: "Trastornos del control de impulsos y tratamiento con antiepilépticos», en Actas Esp Psiquiatr, 2009; 37(4):205-212.

21 AMERICAN PSYCHIATRIC ASSOCIATION: Actualización de la codificación del DSM-5. suplemento del

manual diagnóstico y estadístico de trastornos mentales, Marzo 2014. 
mejor por un trastorno de la conducta, un episodio maníaco o un trastorno de la personalidad antisocial (criterio E) ${ }^{22}$.

Antes de empezar el estudio en profundidad de este trastorno, conviene tener presente que la nueva edición del DSM ha conllevado diferentes cambios respecto al DSM-IV-TR, tanto generales como particulares. En líneas generales podemos decir que el DSM-V, como ECHEBURÚA ${ }^{23}$ destaca, ha eliminado el sistema multiaxial y ha reorganizado los capítulos. Y, aunque lo cierto es que todavía constituye un modelo categorial, hecho que conlleva que cada categoría diagnóstica sea diferente de la salud y del resto de constructos, incorpora un cierto enfoque dimensional, impregnando modestamente con carácter general el manual, especialmente debido a los avances que en campos como la Neurociencia, como seguidamente veremos, se están produciendo, y evitado las «distinciones artificiales» del modelo categorial. En cuanto a la estructura global, de los 17 grandes epígrafes del DSM-IV-TR se pasan a $22^{24}$, incluyendo cambios asociados a la organización de los trastornos dentro del manual, basando su exposición en relación con su aparición en el ciclo vital, esto es, los trastornos del desarrollo primero y los neurocognitivos al final.

No obstante, lo cierto es que su reciente publicación no ha venido exenta de polémica. Autores como Echeburúa cuestionan el aumento de diagnósticos psiquiátricos, la menor exigencia para los criterios diagnósticos en las categorías antiguamente existente ${ }^{25} \mathrm{o}$ la relación entre el grupo encargado de su elaboración y la industria farmacéutica ${ }^{26}$.

Concretamente, en un nuevo capítulo llamado «Trastornos de conducta disruptiva y del control de impulsos», el DSM-V auna los trastornos antes incluidos en el capítulo «Otros trastornos de la infancia, niñez y adolescencia», y en el capítulo "Trastornos del control de impulsos no especificados»; trastornos caracterizados por los problemas en el autocontrol emocional y conductual. Como dato a destacar, y que explica la gran comorbilidad existente entre ambos, el trastorno antisocial de personalidad está incluido también en este capítulo, y, sin duda, hace presagiar una reorganización dimensional de los trastornos en los que, más que a categorías, se valoraran las dimensiones.

22 AMERICAN PSYCHIATRIC ASSOCIATION: Guía de consulta de los Criterios Diagnósticos del DSM-5, 2014

23 ECHEBURÚA, E., SALABERRÍA, K. y CRUZ-SAEZ, M.: «Aportaciones y limitaciones del DSM-5 desde la Psicología Clínica», en Terapia Psicológica, 32(1), 2014.

${ }^{24}$ SANTOS RUIZ, J.L. y SANZ RODRÍGUEZ, L.J.: DSM-5: Novedades y Criterios Diagnósticos, Centro Documentación de Estudios y Oposiciones, 2013.

25 ECHEBURÚA, E., SALABERRÍA, K. y CRUZ-SAEZ, M.: «Aportaciones y limitaciones del DSM-5 desde la Psicología Clínica», en Terapia Psicológica, 32(1), 2014.

26 COSGROVE, L. y KRIMSKY, S.: "A Comparison of DSM-IV and DSM-5 Panel Members' Financial Associations with Industry: A Pernicious Problem Persists», en PLoS Med, 9(3) 2012. 
Desafortunadamente, existen pocos estudios sistemáticos sobre este trastorno, y la mayoría de los trabajos que hacen referencia al mismo se han realizado sobre sujetos que han cometido robos en tiendas o almacenes, no estando en estos casos ni mucho menos claro si tales características corresponden a sujetos con este trastorno o solo es una forma de enmascarar al «ladrón común» y beneficiarse de las atenuaciones de responsabilidad.

Centrándonos en la clínica, en la cleptomanía, el enfermo, de acuerdo con las características que figuran en las clasificaciones internacionales, suele describir una sensación de tensión emocional antes de llevar a cabo la conducta, seguida por una sensación de gratificación o alivio durante o inmediatamente después del acto. El robo no es un hecho planeado, sino que se realiza en solitario, sin la colaboración de terceras personas. $\mathrm{Y}$, aunque generalmente se suele ocultar tales actos, no siempre se tienen en cuenta todas las precauciones para evitar el ser descubierto. Además, aunque la mayoría de los cleptómanos realizan sus robos en tiendas, tampoco se descartan los perpetrados en el ámbito familiar. Aspectos que, sin duda, deben ser tenidos en cuenta en el terreno forense.

La controversia en el abordaje de este trastorno se explica tambien por la falta de datos epidemiológicos fiables, procediendo la información de fuentes de origen distintas, como informes forenses o historias clínicas de pacientes psiquiátricos, especialmente con trastornos de la conducta alimentaria, en los que se desconocen muchos datos interesantes para su abordaje ${ }^{27}$.

Finalmente, se debe destacar que la frecuencia de aparición de este trastorno es del $0,6 \%$ en la población general, aunque algunos autores entienden que sería más frecuente de lo que se venía considerando. El perfil común de la práctica totalidad de los estudios corresponde a una mujer joven que comenzó a robar a los 20. McELROY ${ }^{28}$, autor de los mejores artículos contemporáneos sobre el tema, afirma que un $75 \%$ de los pacientes diagnosticados son mujeres cuyo trastorno tiene una duración media de 16 años, con una frecuencia de 27 robos al mes.

27 McELROY, S.L., KECK, P.E. Jr., POPE, H.G. Jr., HUDSON, J.I., FAEDDA, G.L. y SWANN, A.C.: 1992.

28 McELROY S.L., KECK, P.E. Jr. y PHILLIPS, K.A.: «Kleptomania, compulsive buying, and binge-eating disorder», en J Clin Psyciatry, 1995; 56 Suppl 4:14-26; discussion 27. McELROY S.L., HUDSON, J.I., POPE, H.G. Jr., KECK, P.E. Jr y AIZLEY H.G.: «The DSM-III-R impulse control disorder not elsewhere classified: clinical characteristics and relationship to other psychiatric disorders», en Am J Psychiatry, 1992 Mar; 149 (3):318-27. 


\section{Correlatos neurofisiológicos}

Curiosamente, fue bajo la teoría psicoanalítica desde la que, por primera vez, se empezó a indagar sobre la etiología de la cleptomanía; punto de partida que, posiblemente, ha marcado su tratamiento posterior. Concretamente, el centro de atención de estas pioneras investigaciones fueron los factores psicodinámicos. En este sentido, Wilhelm Stekel, autor psicoanalítico, postuló como explicación de este TCI la conversión en energía cinética de la energía sexual derivada de conflictos edípicos no resueltos por parte de los afectados. Desde entonces, muchos autores han intentado dar una explicación a este problema en base a la posible existencia de conflictos subyacentes. Ahora bien, aunque desde el siglo XX se ha intentado perfilar los caracteres psicológicos característicos de estas personas, como, esencialmente, mujeres con baja socialización ${ }^{29}$, con personalidades depresivas ${ }^{30}$, neuróticas ${ }^{31}$, o psicopáticas, cada vez existe mayor consenso en que no estaríamos ante un único síndrome psicopatológico, siendo sus causas, asimismo, múltiples ${ }^{32}$. En esta línea, el modelo biopsicosocial ${ }^{33}$ engloba esta patología dentro de los llamados Trastornos del Espectro Afectivo, junto a otros trastornos del control de los impulsos, trastornos afectivos, trastornos de ansiedad, y abuso de sustancias psicoactivas ${ }^{34}$.

No es casualidad que algunos autores hayan negado que la cleptomanía sea un trastorno con entidad propia, manifestando que constituiría, en puridad, una simple manifestación clínica dentro del espectro de otro trastorno psiquiátrico. Mantiene esta postura McElroy ${ }^{35}$, que

29 SARASALO, E., BERGMAN, B. y TOTH, J.: «Personality traits and psychiatric and somatic morbidity among kleptomaniacs», en Acta Psychiatr Scand, 1996 Nov; 94 (5):35864.

30 FISHBAIN, D.A.: «Kleptomania as risk-taking behaviour in response to depression», en Am J Psychother, 1987 Oct;41 (4) :598-603.

31 GOSSLING, H.W. y ROSIN, J.: «Kleptomania before and after spontaneous subaracnoid hemorrhage. A neuropsychodynamic case report», en Fortschr Neurol Psychiatr, 1994 May;62 (5) :164-8.

32 CIERPKA, M.: "Psychodynamics of neurotically-induced kleptomania», en Psychiatr Prax, 1986 May;13 (3) :94-103.

33 GOLDMAN, M.J.: "Kleptomania: making sense of the nonsensical», en Am J Psychiatry, 1991 Aug;148 (8) :986-96.

34 McELROY S.L., KECK, P.E. Jr. y PHILLIPS, K.A.: 1995. McELROY S.L., HUDSON, J.I., POPE, H.G. Jr., KECK, P.E. Jr y AIZLEY H.G.: 1992. McELROY, S.L., POPE, H.G. Jr, HUDSON, J.I., KECK, P.E. Jr y WHITE, K.L.: «Kleptomania: a report of 20 cases», en Am J Psychiatry 1991 May; 148 (5):652-7. McELROY S.L., HUDSON, J.I., POPE, H.G. Jr. y KECK, y P.E. Jr.: «Kleptomania: clinical characteristics and associated psychopathology», en Psychol Med, 1991 Feb; 21 (1):93-108. POPE, H.G. Jr. y HUDSON, J.I.: «A supplemental interview for forms of 'affective spectrum disorder'», en Int J Psychiatry Med, 1991; 21 (3):205-32.

35 McELROY S.L., HUDSON, J.I., POPE, H.G. Jr. y KECK, P.E. Jr.: 1991. McELROY S.L., KECK, P.E. Jr. y PHILLIPS, K.A.: 1995, en igual sentido: FISHBAIN, D.A., 1987; POPE, H.G. Jr. y HUDSON, J.I.: 1991. 
consideró que la cleptomanía está relacionada con el espectro de los trastornos afectivos. Además, es interesante destacar como este mismo autor documentó un aspecto interesante: la alta incidencia de trastornos alimentarios entre los pacientes cleptómanos, pues el impulso irresistible para robar, y la excitación experimentada mientras se realiza la conducta estaría en relación con las conductas adictivas ${ }^{36}$. Finalmente, la relación de la cleptomanía con el trastorno obsesivo-compulsivo también se ha defendido, dada la detección de una mayor incidencia de este trastorno en los pacientes con cleptomanía y en sus familiares de primer grado ${ }^{37}$.

A decir verdad, la comorbilidad entre los TCI, los trastornos relacionados con sustancias (TRS) y los trastornos de la personalidad (TPs) es verdaderamente frecuente en la práctica clínica, por lo que, en la actualidad, se continúa cuestionando el carácter independiente de este trastorno $^{38}$. En cualquier caso, como seguidamente se tendrá ocasión de comprobar, esta morbilidad asociada se explica, en parte, por el hecho de que los correlatos neurobiológicos de trastornos como la cleptomanía sean compartidos por el resto de perturbaciones de este grupo, teniendo cada vez más adeptos los partidarios de enfoques dimensionales en el estudio de los trastornos psiquiátricos.

Concretamente, la cleptomanía presenta una alta comorbilidad con el trastorno depresivo mayor, el trastorno obsesivo-compulsivo, el trastorno por estrés postraumático, la bulimia nerviosa y el trastorno de ansiedad generalizada. Esto se explica en base a la concurrencia de diferentes aspectos psicobiológicos comunes entre estos trastornos, como la dimensión impulsiva de la personalidad y determinados neurotransmisores, entre ellos el sistema serotoninérgico, muy importante en las recientes investigaciones.

Adentrándonos, en este punto, en el estudio de su etiología, conviene apuntar que, aunque la investigación sobre los factores biológicos que estarían implicado en la cleptomanía, es muy escasa ${ }^{39}$, partiendo de la relación existente entre la cleptomanía con otros TCI con los que comparte etiología, pueden extenderse los hallazgos respecto a estos. Y precisamente estos estudios abogan por la existencia de un déficit serotonér-

36 McELROY S.L., HUDSON, J.I., POPE, H.G. Jr. y KECK, P.E. Jr.: 1991.

37 McELROY, S.L., POPE, H.G. Jr, HUDSON, J.I., KECK, P.E. Jr y WHITE, K.L.: 1991; McELROY S.L., HUDSON, J.I., POPE, H.G. Jr. y KECK, P.E. Jr.: 1991.

38 CERVERA MARTÍNEZ, G., RUBIO VALLADOLID, G., HARO CORTÉS, G., BOLINCHES CLARAMONTES, F., DE VICENTE MANZANARO, P. y VALDERRAMA ZURIÁN, J.C.: «La comorbilidad entre los trastornos del control de los impulsos, los relacionados con el uso de sustancias y los de la personalidad», en Trastornos Adictivos, Vol. 03. Núm. 01. Enero 2001.

39 MURRAY, J. B.: «Kleptomania: A Review of the Research», en The Journal of Psychology: Interdisciplinary and Applied 1992, 126: 131-7. 
gico en estos pacientes ${ }^{40}$, hecho que explicaría la mejoría de algunos de estos cuando son tratados con fármacos potenciadores de la serotonina (ISRS), los cuales mejoran la neurotransmisión a este nivel.

De todos modos, la hiposerotonergia encontrada en los TCI, que ocasiona que haya una buena respuesta a los fármacos ISRS, no se presenta en todos los trastornos, ni en todos los pacientes del mismo tipo de trastorno, por lo que se sospecha la existencia de una heterogeneidad biológica en los TCI todavía no resuelta que en los próximos años debería ser investigada.

Hoy en día las investigaciones respecto del TCI se centran en la impulsividad ${ }^{41}$. En tal sentido, la principal alteración neurobiológica encontrada en la literatura en el TCI es el déficit serotoninérgico ${ }^{42}$. En estudios recientes se describe que existirían, asimismo, alteraciones en otros sistemas de neurotransmisión ${ }^{43}$, como son la hiperactividad noradrenérgica, la disfunción dopaminérgica y colinérgica, un incremento de testosterona y endorfinas, una hipofunción del eje hipotálamo-hipofisosuprarrenal, una hipofunción gabaérgica y una hiperfunción glutamatérgica $^{44}$. Ello es compatible con la documentada experiencia clínica según la cual, fármacos como los antiepilépticos, sin acción serotoninérgica, son también efectivos en las conductas impulsivas. Así pues, la participación de otros mecanismos neurobiológicos ${ }^{45}$, junto con alteraciones genéticas y neuroanatómicas, podrían estar relacionadas con una fragilidad neurológica congénita o postraumática ${ }^{46}$. Desafortunadamente, como

40 Aunque en la cleptomanía no se cuentan con datos, si en piromanía y ludopatía. Para más información; VIRKUNNEM, M., NUUTILA, A., GOODWIN, F.K. y LINNOILA, M.: "Cerebrospinal fluid monoamine metabolite levels in male arsonists», en Arch Gen Psychiatry. 1987 Mar; 44(3):241-7.

${ }_{41}$ RONCERO, C., RODRÍGUEZ-URRUTIA, A., GRAU-LÓPEZ L. y CASAS, M.: 2009.

42 PALLANTI, S. y HOLLANDER, E.: "Pathological gambling», en HOLLANDER, E. (ed.) Clinical Manual of Impulse Control Disorders. Washington: American Psychiatric Publishing, 2006. ROS, S., DIEZ, B. y CASANOVA, N.: "Anticonvulsivantes en el tratamiento de la impulsividad», en Actas Esp Psiquiatr, 2008; 36 (Suppl. 3):46-62.

43 KALENSCHER, T., OHMANN, T. y GUNTURKUN, O.: "The neuroscience of impulsive and self-controlled decisions», en Int J Psychophysiol, 2006; 62(2):203-11.

44 ROS, S., DIEZ, B. y CASANOVA, N.: 2008.

45 Respecto a los estudios farmacológicos, conviene tener presente que existen estudios, como el de McElroy, en los que se demuestra que el litio en combinación con fluoxetina ha sido eficaz. No obstante, este mismo autor también ha comprobado que el tratamiento con carbamazepina, encombinación con clomipramina no mejora los síntomas de cleptomanía. Y en el estudio de Dannon se verificó una buena respuesta a topiramato a dosis de hasta $150 \mathrm{mg}$, solo o junto con fluoxetina. Referencias: McELROY S.L., HUDSON, J.I., POPE, H.G. Jr. y KECK, P.E. Jr., 1991. McELROY, S.L., POPE, H.G. Jr, HUDSON, J.I., KECK, P.E. Jr y WHITE, K.L., 1991. DANNON, P.N. «Topiramate for the treatment of kleptomania: a case series and review of the literature», en Clin Neuropharmacol, 2003; 26(1):1-4.

46 ELLIOTT, F.A.: «Neurological findings in adult minimal brain dysfunction and the dyscontrol syndrome», en J Nerv Ment Dis, 1982; 170(11):680-7. 
previamente se apuntó, no hay estudios biológicos específicos sobre la cleptomanía ni sobre la piromanía.

Esta situación comporta que el tratamiento de estos trastornos sea muy complejo, pues las dificultades en esclarecer la etiopatogenia de las conductas impulsivas y la muy frecuente existencia de comorbilidad complica en gran medida las cosas. De hecho, es probable que se trate de un conjunto de entidades relacionadas clínicamente entre sí, pero con distinta base neurobiológica. Y, precisamente el avance en la investigación neurocientífica puede permitirnos un mejor conocimiento de los distintos mecanismos implicados en estos trastornos.

De entrada, existe suficiente evidencia para afirmar que la función serotoninérgica estaría alterada. No en balde, como se destacó en líneas anteriores, han sido administrados satisfactoriamente fármacos con acción sobre este sistema, aunque, en realidad, el tratamiento con estos medicamentos es insuficiente. En cualquier caso, teniendo en cuenta el éxito del tratamiento antidepresivo con ISRS, se postula que podría ser este el neurotransmisor implicado principalmente en los trastornos del espectro afectivo y, más concretamente, en los trastornos del control de los impulsos como la cleptomanía. En efecto, la evidencia de que los fármacos antipsicóticos atípicos actúan, además de sobre receptores dopaminérgicos, en los serotonérgicos, podría explicar la asociación conmórbida con patologías como la piromanía ${ }^{47}$, o inclusive con la esquizofrenia ${ }^{48}$, celotipia ${ }^{49}$, y otros estados psicóticos ${ }^{50}$. Tampoco cabe olvidar la relación de la cleptomanía con el alcoholismo, especialmente respecto de patologías sexuales como el exhibicionismo ${ }^{51}$, o, inclusive, alteraciones en otros sistemas de neurotransmisión ${ }^{52}$.

47 PIERLOOT R.A., WELLENS, W. y HOUBEN, M.E.: «Elements of resistance to a combined medical and psychotherapeutic program in anorexia nervosa. An overview», en Psychother Psychosom, 1975; 26(2):101-17.

${ }_{48}$ DE MONTJOYE, B.V., WAMBERGUE, D., LECAMP, M. y LAROME, A.: «Kleptomania and pyromania. Apropos of a case», en Ann Med Psychol (Paris) 1992 Dec; 150 (10):734-8.

49 CHONG, S.A.: «Schizophrenia and kleptomania: a case report. A biological link?» en Ann Acad Med Singapore, 1995 Nov; 24 (6):860-2.

50 RIKOVSKY, S., STROKY, H. y WIDERMANNOVA, L.: «Kleptomania and pathic possession», en Cesk Psychiatr, 1968 Oct; 64 (5):326-36. KYSHTOBAEVA, ZSH.: "Psychopathology and clinical picture of impulsive tendencies during schizophrenia», en Zh Nevropatol Psikhiatr 1987; 87 (9):1383-7.

51 LEJOYEUX, M., FEUCHE, N., LOI, S., SOLOMON, J. y ADES J.: «Study of impulse-control disorders among alcohol-dependent patients», en J Clin Psychiatry, 1999 May; 60 (5):302-5.

52 RONCERO, C., RODRÍGUEZ-URRUTIA, A., GRAU-LÓPEZ L. y CASAS, M.: 2009. 


\section{ESTUDIO JURISPRUDENCIAL}

\section{Tratamiento del TS}

Para empezar, cabe destacar que no existe una doctrina consolidada por el TS sobre la incidencia de la cleptomanía en la imputabilidad. Esto se explica, junto con las controversias que envuelven a esta categoría doctrinal, por el escaso número de casos en los que este tribunal se ha tenido que pronunciar sobre su apreciación, pues de normal no se encuentran recursos de casación en los que se discuta este trastorno en relación con los hechos delictivos, debido al escaso valor de lo sustraído por las personas que padecen este trastorno ${ }^{53}$.

En cualquier caso, se entiende que la cleptomanía puede ser considerada una patología psíquica incardinable en los términos «anomalía o alteración psíquica» que figuran en la eximente del art. $20.1 \mathrm{CP}$, por lo que, si los hechos se producen durante el padecimiento del impulso que en las recientes investigaciones de constata, podría ser de aplicación tanto la eximente completa, como la incompleta o la atenuante analógica, dependiendo de la afectación sobre la capacidad de actuar ${ }^{54}$.

No obstante, no se ha encontrado ningún caso en el que el TS haya aplicado la eximente completa de anomalía o alteración psíquica debido a una cleptomanía, pues lo cierto es que en la mayoría de casos se deniega cualquier tipo de rebaja penal. Esta fue la respuesta en uno de los escasos casos en los que el TS se ha pronunciado al respecto, un supuesto en el cual, si bien niega cualquier tipo de repercusión a este trastorno, efectúa una aproximación al mismo verdaderamente interesante, que recoge algunas de las últimas líneas de investigación respecto de este trastorno.

En su sentencia núm. 569/2012, de 27 junio, el TS estimó inapreciable la eximente del artículo $20.1 \mathrm{CP}$, en relación con una supuesta cleptomanía, en base a los hechos probados en el proceso. En el mismo se enjuició el caso de un empleado de una asesoría jurídica, acusado de un delito continuado de falsedad en documento mercantil. La citada asesoría cobraba una cantidad mensual por los servicios de asesoría legal prestados a una clínica dental, pero con la finalidad de obtener un enriquecimiento ilícito el acusado convenció a los responsables de la Clínica para recibir de ésta, en efectivo o mediante cheques al portador, el dinero que la empresa había de abonar periódicamente a la Agencia Tributaria en concepto de pagos de IRPF e Impuesto de Sociedades, con el compromiso de hacer los pagos correspondientes. Sin embargo, el acusado no ingresó estos pagos en la Agencia Tributaria, quedándoselo para

\footnotetext{
53 Así lo reconoce la SAP de Sevilla 697/2003, de 23 de diciembre.

54 URRUELA MORA, A.: 2004, p. 329.
} 
uso propio. Ante tales hechos, el tribunal entendió que la premeditación manifestada por el acusado, y las argucias usadas para hacerse con esta cuantiosa suma de dinero, eran incompatibles con la fenomenología de este trastorno.

Concretamente, en base al informe pericial médico aportado a juicio y las explicaciones ofrecidas por el perito, estimó que no existía suficiente evidencia para afirmar la existencia de cleptomanía en el acusado, ni que ésta tuviera intensidad suficiente como para anular o limitar las capacidades intelectivas y volitivas del mismo. En este sentido, el perito declaró que «la cleptomanía era un trastorno de control de impulsos que parece incompatible con la premeditación», puesto que se trata de un comportamiento impulsivo. Como se destaca, la cleptomanía se caracteriza por las conductas reiteradas de ceder a los impulsos y por la tensión, a veces intolerable, de sustraer objetos que no son obtenidos por su valor monetario o como ganancia o beneficio patrimonial. No obstante, en el presente caso el acusado fue selectivo en cuanto a los medios utilizados para cometer su actuación, como también para la elección de la víctima. Asimismo, pone de manifiesto la reiteración delictiva, con el uso de medios fraudulentos para encubrir y justificar su acción.

Es destacable igualmente como el TS se hace eco de los últimos estudios etiológicos respecto de esta categoría, al afirmar que «desde el punto de vista de la fenomenología, no es descartado pensar que la cleptomanía sea tan solo una manifestación clínica concreta en la que subyace un trastorno obsesivo compulsivo. Y ello es así porque el verdadero trastorno de cleptomanía lleva aparejada la conciencia de que es un acto absurdo, sin sentido racional, tras el que se experimenta bienestar y liberación por la desaparición de la tensión, pero también autoreproches o ideas de culpa, es decir, con malestar clínico significativo (FD $7^{\circ}$ )». Además, destacó otro aspecto a considerar, como son los delitos en relación con la definición específica del trastorno, como son los hurtos y robos de diversos objetos en tiendas cometidos de forma impulsiva, los cuales no se deberían confundir con los hurtos en tiendas para satisfacer caprichos, o la venta posterior de los artículos sustraídos para la supervivencia.

\section{Tratamiento de las Audiencias Provinciales}

De igual manera que el TS, las Audiencias Provinciales (en adelante, AAPP) tampoco suelen pronunciarse frecuentemente respecto de la apreciación de la cleptomanía. Y, cuando lo hacen, suelen negar la atenuación de responsabilidad en base a un trastorno que, en ocasiones, se utiliza para justificar las conductas de simples ladrones habituales. No obstante, cabe destacar dos sentencias de AAPP en las que se ha apreciado esta eximente completa. 
En primer lugar, la Audiencia Provincial (en adelante, AP) de Alicante $^{55}$ reconoció este trastorno, junto con una ludopatía, como causa de la anulación de la capacidad volitiva de la acusada, en su sentencia núm. 57/1998, seguramente debido a que la mujer, en el momento de los hechos, estaba sometida a tratamiento. También reconoció la eximente completa la AP de Sevilla ${ }^{56}$, efectuando un interesante análisis del trastorno en su pronunciamiento. La audiencia destaca como el impulso en cuestión llega de manera inesperada, sin planearlo previamente, y resulta irrefrenable para el enfermo, el cual, aunque es consciente en todo momento de la antijuridicidad de su conducta, no es capaz de controlar su impulso, actuando en solitario. No obstante, conviene destacar que en este caso la acusada padecía un retraso mental leve; cuadro que explica, en parte, la apreciación de esta eximente completa en un robo de bisutería en unos grandes almacenes. Con independencia de ello, resulta conveniente destacar las afirmaciones de la audiencia, al entender, de forma apropiada, que conforme a la caracterización de la cleptomanía que figura en las clasificaciones internacionales, el cleptómano no es penalmente imputable por los pequeños hurtos o robos que cometa bajo el impulso de su trastorno, como se produjo en ese caso, ya que en ese momento no era accesible a la motivación por la norma penal, pues estaba dominado por un impulso que todos los autores describen como «irresistible o irrefrenable» de cometer la acción antijurídica, con el objetivo de aliviar la tensión insoportable padecida como consecuencia del trastorno.

Por otro lado, también encontramos la aplicación de la eximente incompleta. Esta fue la apreciada por la SAP de Madrid 138/2003, de 7 de marzo, al entender que la cleptomanía que padecía la acusada disminuía únicamente su capacidad volitiva, o la SAP de Barcelona de 30 de abril de 2001, en un caso en la que se ejemplifica la comorbilidad existente analizada en este trastorno. Concretamente, la acusada padecía, junto a la cleptomanía, un trastorno mixto de la personalidad, depresión y politoxicofilia, apreciando la Audiencia que esta mezcla de trastornos provocó en la acusada una limitación parcial de sus facultades volitivas. En la misma línea, la AP de Huesca, en su sentencia 88/2001, de 16 de mayo, reconoció una disminución en las facultades mentales de la acusada, debido a la cleptomanía padecida ante una situación ansiosa, si bien en este supuesto se apreció la circunstancia atenuante analógica.

En cualquier caso, como se avanzó, la regla general es la apreciación de la imputabilidad plena, negando cualquier tipo de repercusión. Como ejemplo de estos pronunciamientos podemos destacar la SAP de Pontevedra 6/2002, de 22 de abril, en al supuesto de un robo en casa habitada a la que accede tras violentar la puerta de entrada con una pala, o la SAP de Madrid 345/2011 de 28 julio, ante un delito continuado de falsedad

55 SAP de Alicante 57/1998, de 2 febrero.

56 SAP de Sevilla 697/2003, de 23 de diciembre. 
en documento mercantil, al corroborarse en estos casos la ausencia de relación del delito cometido con el trastorno alegado por los acusados, puesto que el mismo es imprescindible para apreciar una atenuación o exclusión de la responsabilidad.

La inexistencia de la citada relación de causalidad entre el trastorno padecido y los hechos perpetrados por el acusado fue el motivo por el que la AP de Ávila rechazó la aplicación de la eximente del art. $20.1 \mathrm{CP}$ en uno de los últimos pronunciamientos sobre cleptomanía por parte de nuestros tribunales. En su sentencia núm. 111/2019, de 22 noviembre, la Audiencia se manifestó respecto de la apreciación de este trastorno en la acusada de una estafa en un establecimiento comercial del que era clienta, y destacó que, «aun evidenciándose su realidad, lo cierto es que, conforme a la pericial verificada por el Médico Forense en el acto de la vista oral, la misma se traduce en actos impulsivos de cleptomanía, pero no le impide ni influye en otros aspectos de su vida ni, tampoco, en la ideación de un plan más elaborado para la consecución de los fines que persigue, ya que el diagnóstico que presenta se traduce únicamente en conductas de hurto compulsivo, pero son sólo esas las conductas en las que se predica dicho trastorno, sin que ello le impida observar un comportamiento voluntario de ideación, ni que el mismo se forme de manera anormal en otros aspectos de su vida, siendo perfectamente capaz de llevarlo a ejecución (FD 4')»; como estimó precisamente que ocurrió en el presente supuesto.

\section{REFLEXIONES FINALES}

Tras el estudio efectuado, se advierte que la cleptomanía es una categoría diagnóstica fuertemente discutida en el ámbito clínico, lo que dificulta la existencia de un tratamiento jurisprudencial uniforme. En primer lugar, en base al análisis de la cleptomanía desde una perspectiva clínica, especialmente centrada en los últimos avances que desde la Neurociencia cognitiva se desarrollan al respecto, se puede concluir que la misma es un trastorno en el área de la impulsividad que impide a los sujetos resistir los impulsos que sienten de robar objetos. Esta caracterización permite, siempre que el delito en cuestión consista en pequeños hurtos sin premeditación, que los tribunales puedan apreciar, en función de su intensidad, tanto la eximente completa del art. $20.1 \mathrm{CP}$, como la eximente incompleta del art. $21.1 \mathrm{CP}$ en relación con el anterior, junto con la circunstancia atenuante analógica, cuando los acusados presenten un trastorno de cleptomanía.

Asimismo, como previamente se apuntó, los adelantos en el campo neurocientífico pueden ser útiles en la validación de trastornos del control de los impulsos, pues pueden permitir, concretamente respecto de la cleptomanía, la distinción de aquellos sujetos que sufren esta patología de los delincuentes habituales, pues la alegación de la cleptomanía en 
algunos procesos no es más que un recurso que pretende una simple reducción de la condena, confundiéndose sus notas definitorias con las del simple delincuente común.

No obstante, lo cierto es que las últimas investigaciones apuntan a que muchas de las categorías diagnosticas imperantes en la actual nosología psiquiátrica responden a una diferenciación artificial en la que intervienen las mismas bases neurobiológicas. En esta línea, la etiología de los TCI, TPs y TRS parece que sería la misma, teniendo como nota central la impulsividad que, como es de sobra conocido, los sujetos que padecen estos trastornos presentan. Por ello, los especialistas que apuestan por una configuración dimensional de los trastornos, entienden que los mismos deberían quedar englobados en una misma dimensión. De todos modos, lo cierto es que, al margen de estas discusiones respecto de su concreta etiqueta diagnostica, en el proceso penal la clave descansa en la incidencia del trastorno en las capacidades cognoscitivas generales, conforme a la fórmula mixta que nuestro $\mathrm{CP}$ acoge; una afectación que está siendo validada y que podría ser graduada, como supra se ha destacado, en atención a la incidencia y gravedad del trastorno en el supuesto en cuestión.

Pues bien, pese a no existir una doctrina consolidada del TS respecto de estos trastornos, debido principalmente a la escasa trascendencia de las actuaciones delictivas de estos sujetos, el tratamiento jurisprudencial de la cleptomanía, especialmente por parte de las AAPP, ha mejorado con el transcurso de los años, y su repercusión en la imputabilidad va perfilándose de forma adecuada. Uno de los puntos más complicados es, sin duda, la distinción de estos supuestos de los casos en los que nos encontramos ante un simple delincuente habitual. Y, respecto de esta complicada distinción, los estudios neurobiológicos pueden ser de especial utilidad, dado que permitirán la compleja delimitación de los contornos de este trastorno y su incidencia en la psique de los acusados.

Siguiendo la SAP de Sevilla núm. 697/2003, de 23 de diciembre, antes vista, se puede concluir que el cleptómano no debería ser penalmente imputable por los pequeños hurtos o robos que cometa bajo el impulso de su trastorno, pues en esos casos no es accesible a la motivación por la norma penal, ya que, a pesar de conocer la ilicitud del hecho, no puede actuar de acuerdo a dicha comprensión. Ahora bien, los tribunales deberían valorar de forma cuidadosa tanto la relación del trastorno con la conducta en cuestión, como la gravedad de este y su incidencia en la capacidad de control del acusado, pues únicamente en supuestos de excepcional gravedad puede llegar a plantearse la apreciación de una eximente completa.

De todos modos, como se ha podido comprobar en el análisis jurisprudencial efectuado, la regla general por parte de los tribunales es la apreciación de la imputabilidad plena en estos casos, teniendo en cuenta que en numerosas ocasiones se confunde este trastorno con las notas 
definitorias del simple delincuente común, como precisamente ocurre en la SAP de Ávila núm. 111/2019, de 22 de noviembre. Asimismo, en los escasos supuestos en los que se ha llegado a reconocer la relación de causalidad entre la cleptomanía y los concretos actos delictivos, este trastorno presenta comorbilidad con diferentes perturbaciones con las que comparte etiología según los modelos dimensionales, como los trastornos del espectro afectivo.

\section{BIBLIOGRAFÍA}

American Psychiatric Association, Actualización de la codificación del DSM-5. Suplemento del manual diagnóstico y estadístico de trastornos mentales. Marzo 2014.

- Diagnostic and Statistical Manual of Mental Disorders (DSM-III) 1980.

— Diagnostic and Statistical Manual of Mental Disorders (DSM-V) 2014.

Blanco Lozano, C.: «El concepto penal de imputabilidad», en La Ley, 2002-1' ${ }^{\circ}$ D-18.

Cabrera Forneiro, J. y Fuertes Rocañin, J.C.: La Salud Mental en los Tribunales. Manual de Psiquiatría Forense y Deontología Profesional, $2^{\mathrm{a}}$ ed. revisada y ampliada, Arán, Madrid, 2007.

Cervera Martínez, G., Rubio Valladolid, G., Haro Cortés, G., Bolinches Claramontes, F., De Vicente Manzanaro, P. Y Valderrama ZURIÁN, J.C.: «La comorbilidad entre los trastornos del control de los impulsos, los relacionados con el uso de sustancias y los de la personalidad», en Trastornos Adictivos, Vol. 03. Núm. 01. Enero 2001.

CHong, S.a.: «Schizophrenia and kleptomania: a case report. A biological link?» en Ann Acad Med Singapore, 1995 Nov; 24 (6):860-2.

CIERPKA, M.: «Psychodynamics of neurotically-induced kleptomania», en Psychiatr Prax, 1986 May;13 (3) :94-103.

Cobo Del Rosal, M. y Vives Antón, T.S.: Derecho Penal. Parte general, $5^{\text {a }}$ ed. corregida, aumentada y actualizada, Tirant lo Blanch, Valencia, 1999, p. 576.

Cosgrove, L. y Krimsky, S.: «A Comparison of DSM-IV and DSM-5 Panel Members' Financial Associations with Industry: A Pernicious Problem Persists», en PLoS Med, 9(3) 2012.

DANNON, P.N.: «Topiramate for the treatment of kleptomania: a case series and review of the literature», en Clin Neuropharmacol, 2003; 26(1):1-4. 
De Montjoye, B.v., Wambergue, D., Lecamp, M. Y Larome, A.: «Kleptomania and pyromania. Apropos of a case», en Ann Med Psychol (Paris) 1992 Dec; 150 (10):734-8.

Echeburúa Odriozola, E., Amor Andrés, P.J. Y Yuste García, J.: «Atenuación de la responsabilidad penal en la ludopatía: Bases psicopatológicas», en Psicopatología Clínica Legal y Forense, Vol. 1, $N^{\circ}$ 0, 2000, p. $59-76$.

Echeburúa, E., Salaberría, K. Y CruZ-Saez, M.: «Aportaciones y limitaciones del DSM-5 desde la Psicología Clínica», en Terapia Psicológica, 32(1), 2014.

ELLIOTT, F.A.: «Neurological findings in adult minimal brain dysfunction and the dyscontrol syndrome», en J Nerv Ment Dis, 1982; 170(11): 680-7.

EsBEc, E. Y Echeburrua, E.: «La reformulación de los trastornos de la personalidad en el DSM-V», en Actas Esp Psiquiatría, 39 (1), 2011, pp. 1-11.

FISHBAIN, D.A.: «Kleptomania as risk-taking behaviour in response to depression», en Am J Psychother, 1987 Oct;41 (4) :598-603.

Gimbernat ORDEIG, E.: ¿Tiene un futuro la Dogmática jurídico-penal?, en Estudios de Derecho Penal, $3^{\mathrm{a}}$ ed., Madrid, Tecnos, 1990, p. 157.

Gisbert Calabuig, J.A., Sánchez Blanque, a. y Castellano Arroyo, M.: «Neurosis», en GisBert CALABUig, J.A: Medicina Legal y Toxicología, $6^{\mathrm{a}}$ Edición, Masson, 2004, p. 1179.

Goldman, M.J.: «Kleptomania: making sense of the nonsensical», en Am J Psychiatry, 1991 Aug;148 (8):986-96.

GossLING, H.W. y Rosin, J.: «Kleptomania before and after spontaneous subaracnoid hemorrhage. A neuropsychodynamic case report», en Fortschr Neurol Psychiatr, 1994 May;62 (5):164-8.

Homs Sanz De La Garza, J.: Avances en medicina legal: Ingeniería genética, alteraciones psíquicas y drogas, J.M. Bosch, 1999, p. 53.

IBÁÑEz CuADRADO, A., y SAIZ RuIZ, J.: «Trastornos de los hábitos y del control de los impulsos», en Tratado de Psiquiatría, cap. 32, p. 579 - 602.

Kalenscher, T., OHMANN, T. Y GunTURKun, O.: «The neuroscience of impulsive and self-controlled decisions», en Int J Psychophysiol, 2006; 62(2):203-11.

KyshtoBAEVA, ZSH.: «Psychopathology and clinical picture of impulsive tendencies during schizophrenia», en Zh Nevropatol Psikhiatr 1987; 87 (9):1383-7.

Lamote, H., Tan, K.L. y Verhoeven, W.M.: «Frontotemporal dementia in a young woman with apparent schizophrenia», en Ned Tijdschr Geneeskd, 1998 Aug 29; 142 (35):1962-5. 
Lejoyeux, M., Feuche, N., Loi, S., Solomon, J. y Ades J.: «Study of impulse-control disorders among alcohol-dependent patients», en J Clin Psychiatry, 1999 May; 60 (5):302-5.

Martínez Garay, L. La imputabilidad penal. Concepto, fundamento, naturaleza jurídica y elementos, Valencia, Tirant lo Blanch, 2005, p. 15.

McElroy S.l., Hudson, J.I., Pope, H.G. JR. y Keck, y P.E. Jr.: «Kleptomania: clinical characteristics and associated psychopathology», en Psychol Med, 1991 Feb; 21 (1):93-108.

Mcelroy S.L., Hudson, J.I., Pope, H.G. JR., Keck, P.E. JR Y Aizley H.G.: «The DSM-III-R impulse control disorder not elsewhere classified: clinical characteristics and relationship to other psychiatric disorders», en Am J Psychiatry, 1992 Mar; 149 (3):318-27.

McElroy S.L., Keck, P.E. JR. Y PhILliPS, K.A.: «Kleptomania, compulsive buying, and binge-eating disorder», en J Clin Psyciatry, 1995; 56 Suppl 4:14-26; discussion 27.

McElroy, S.L., Keck, P.E. JR., Pope, H.G. JR., Hudson, J.I., Faedda, G.L. Y SWANN, A.C.: "Clinical and research implications of the diagnosis of dysphoric or mixed mania or hypomania», en Am J Psychiatry. 1992 Dec; 149(12):1633-44.

McElroy, S.L., Pope, H.G. JR, Hudson, J.I., Keck, P.E. JR Y White, K.L.: «Kleptomania: a report of 20 cases», en Am J Psychiatry 1991 May; 148 (5):652-7.

Mir Puig, S.: Derecho Penal. Parte General, $7^{\text {a }}$ ed., $3^{\text {a }}$ reimpr., Reppertor, Barcelona, 2006, p. 558.

Morales Prats, F. (coordinador) y Quintero Olivares, G.: Comentarios al nuevo Código Penal, $4^{\mathrm{a}}$ ed., Thomson-Aranzadi, Navarra, 2005, p. 148.

Muñoz Conde, F. y García Arán, M.: Derecho Penal. Parte General, 6a ed., rev. y puesta al día, Tirant lo Blanch, Valencia, 2004, p. 372.

MURRAY, J. B.: «Kleptomania: A Review of the Research», en The Journal of Psychology: Interdisciplinary and Applied 1992, 126: 131-7.

Pallanti, S. y Hollander, E.: «Pathological gambling», en HOLLANDER, E. (ed.) Clinical Manual of Impulse Control Disorders. Washington: American Psychiatric Publishing, 2006.

Pierloot R.A., Wellens, W. y Houben, M.E.: «Elements of resistance to a combined medical and psychotherapeutic program in anorexia nervosa. An overview», en Psychother Psychosom, 1975; 26(2):101-17.

POPE, H.G. JR. Y Hudson, J.I.: «A supplemental interview for forms of 'affective spectrum disorder'», en Int J Psychiatry Med, 1991; 21 (3):205-32. 
Rikovsky, S., Stroky, H. y Widermannova, L.: «Kleptomania and pathic possession», en Cesk Psychiatr, 1968 Oct; 64 (5):326-36.

Roncero, C., Rodríguez-Urrutia, A., Grau-López, L., Casas, M.: «Trastornos del control de impulsos y tratamiento con antiepilépticos», en Actas Esp Psiquiatr, 2009; 37(4):205-212.

Ros, S., Diez, B. Y Casanova, N.: «Anticonvulsivantes en el tratamiento de la impulsividad», en Actas Esp Psiquiatr, 2008; 36 (Suppl. 3):46-62.

Santos Ruiz, J.L. y Sanz Rodríguez, L.J.: DSM-5: Novedades y Criterios Diagnósticos, Centro Documentación de Estudios y Oposiciones, 2013.

Sarasalo, E., Bergman, B. Y Toth, J.: «Personality traits and psychiatric and somatic morbidity among kleptomaniacs», en Acta Psychiatr Scand, 1996 Nov; 94 (5):358-64.

UrRuela Mora, A.: Imputabilidad penal y anomalía o alteración psíquica. La capacidad de culpabilidad penal a la luz de los modernos avances en psiquiatría y genética, Comares, Bilbao-Granada, 2004, p. 156.

Virkunnem, M., NuUtila, A., Goodwin, F.K. Y Linnoila, M.: «Cerebrospinal fluid monoamine metabolite levels in male arsonists», en Arch Gen Psychiatry. 1987 Mar; 44(3):241-7.

\section{JURISPRUDENCIA}

SAP de Alicante 57/1998, de 2 de febrero.

SAP de Huesca, 88/2001, de 16 de mayo.

SAP de Pontevedra, 6/2002, de 22 de abril.

SAP de Madrid, 138/2003, de 7 de marzo.

SAP de Sevilla 697/2003, de 23 de diciembre.

SAP de Sevilla 697/2003, de 23 de diciembre.

SAP de Madrid, 345/2011, de 28 de julio.

SAP de Ávila, 111/2019, de 22 noviembre.

STS 1400/1999 de 9 de octubre.

STS 314/2005, de 9 de marzo.

STS 175/2008, de 14 de mayo.

STS 569/2012, de 27 de junio. 